\title{
Isolated Ocular Sarcoidosis Mimicking Ring Melanoma
}

\author{
Carsten Faber ${ }^{\mathrm{a}}$ Rasmus Ejstrup ${ }^{\mathrm{a}}$ Karine Madsen $^{\mathrm{b}}$ Steffen Heegaard ${ }^{\mathrm{a}, \mathrm{c}}$ \\ David Scheie $^{c}$ Jens Folke Kiilgaard ${ }^{a}$ \\ aDepartment of Ophthalmology, University Hospital Copenhagen, Rigshospitalet, Copenhagen, Denmark; \\ ${ }^{b}$ Department of Clinical Physiology, Nuclear Medicine and PET, University Hospital Copenhagen, \\ Rigshospitalet, Copenhagen, Denmark; ' Department of Pathology, University Hospital Copenhagen, \\ Rigshospitalet, Copenhagen, Denmark
}

\section{Established Facts}

- Ring melanoma is very rare, and the diagnosis may be elusive.

- Treatment of a ring melanoma is destructive and often includes enucleation.

- Sarcoidosis may be isolated to the eye and present with granulomas in the anterior segment of the eye.

\section{Novel Insights}

- Ocular sarcoidosis may mimic all clinical features of a ring melanoma.

- Biopsy is necessary before destructive surgery is carried out.

- A subtenon injection of triamcinolone may be sufficient to treat isolated ocular sarcoidosis.

\section{Keywords}

Ocular sarcoidosis · Inflammation - Uveal melanoma .

Diagnostics $\cdot$ Transscleral biopsy

\begin{abstract}
We report the case of a 25 -year-old female, who presented with a large, pale tumor of her ciliary body that extended to $>180^{\circ}$ of the anterior chamber angle. All preoperative examinations including ultrasound biomicroscopy, scleral transillumination, and MRI indicated melanoma. A thorough systemic work-up was negative. A diagnosis of ring melanoma was suspected, and the patient was scheduled for
\end{abstract}

\section{KARGER}

(๑) 2019 S. Karger AG, Basel

karger@karger.com

www.karger.com/oop enucleation. However, perioperative frozen section indicated granulomatous inflammation. The enucleation was cancelled, and a subtenon injection of triamcinolone was administered, which resulted in the disappearance of the tumor. Together, the findings meet the criteria for the diagnosis of isolated ocular sarcoidosis. This case demonstrates that a sarcoid granuloma can mimic all clinical features of a ring melanoma. Therefore, a biopsy should be done before destructive surgery is carried out.

(c) 2019 S. Karger AG, Basel

This case was previously presented at the OOG 2019 meeting in London, UK. 

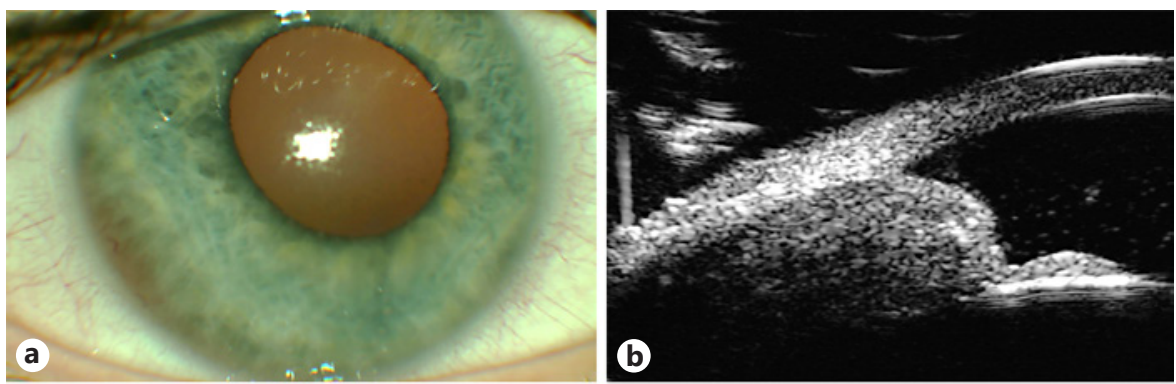

Fig. 1. A 25-year-old woman in general good health presented with large, corneal precipitates and a grey, vascular tumor extending between the 2 and 11 o'clock position of the angle (a). On ultrasound biomicroscopy, the tumor extended posteriorly to the ciliary body and demonstrated homogeneous, medium internal reflectivity (b). On MRI, the signal from the tumor was low on T2- (c) but high on T1-weighted images (d) with enhancement of gadolinium (e). On 18-FDG HRRT PET fused with MRI, the tumor showed high uptake (f).
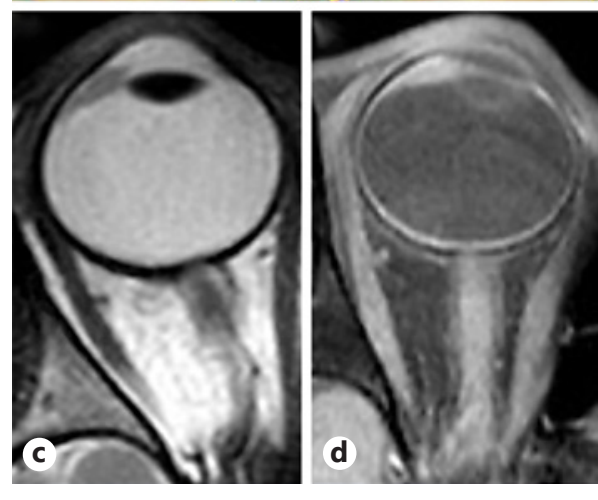

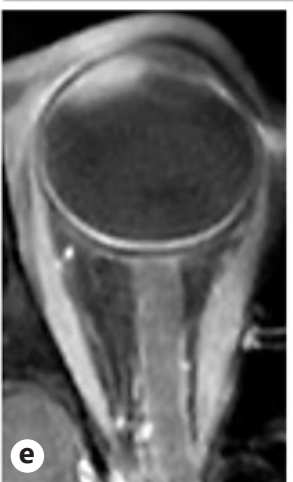

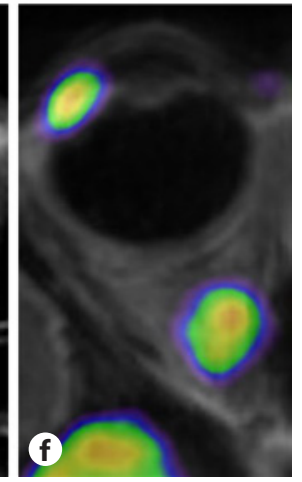

\section{Case Presentation}

A 25-year-old woman was admitted due to redness and blurred vision of her right eye. She did not complain about photophobia, floaters, or pain. The patient was in general good health and did not have a previous medical history.

On examination of her right eye, a best-corrected visual acuity of 0.9 was noted, and the intraocular pressure was $25 \mathrm{~mm} \mathrm{Hg}$. Slitlamp examination revealed mutton-fat precipitates on the lower part of the cornea and in the inferotemporal part of the iris, and in the chamber angle, a large, grey tumor with prominent vessels was noted (Fig. 1). On gonioscopy, the tumor extended from the 2 to 11 o'clock position of the trabecular meshwork. On scleral transillumination, the tumor demonstrated a blockage. Using ultrasound biomicroscopy, the tumor was found to be continuous with the ciliary body and demonstrated hollowness with a homogeneous, medium internal reflectivity. On MRI, the tumor measured $3 \times$ $8 \mathrm{~mm}$ axially. The tumor had a low signal on T2-weighted images and a high signal with gadolinium enhancement on T1-weighted images. A regional high resolution 18F-FDG HRRT PET scan showed high metabolic activity in the tumor, while a whole-body 18F-FDG PET/CT revealed no other pathological findings. Fundoscopy was unremarkable, and the left eye was normal. Extensive laboratory examinations were negative including s-ACE, soluble IL-2 receptor, quantiferon, syphilis, and ANCA.

Based on the above findings, the diagnosis of an amelanotic ring melanoma with masquerading inflammation was suspected, and the patient was scheduled for biopsy and enucleation. A transscleral technique was used to obtain the biopsy. In brief, the sclera was cut with a diamond knife, and a scleral lamellar flap was created using a razor blade to gain access to the anterior chamber. First, it was attempted to obtain tissue for cytology using a $25 \mathrm{G}$ vitrector, but due to sparse material, an excisional biopsy was ob- tained from the peripheral part of the exposed tumor using Vanna's scissors. Perioperative frozen section indicated granulomatous inflammation of the tissue. Therefore, enucleation was cancelled, and a subtenon injection of $40 \mathrm{mg}$ triamcinolone (Kenalog) was administered. Later, histology showed a non-necrotizing granulomatous inflammation. Immunohistochemistry demonstrated CD3-, CD20-, and CD68-positive cells in the granuloma (Fig. 2). Extensive staining was negative for signs of infection (CMV, spirochete, fungi, Ziehl-Neelsen, and Gram) and malignancy (MelanA, cytokeratins, OCT3/4, Sall4, CD1A, Langerin, CD30, and PAX8).

The patient was treated with topical dexamethasone and chloramphenicol for 4 weeks. During the following 2 months, the tumor along with the symptoms gradually disappeared, and visual acuity normalized (Fig. 2). At follow-up after 9 months, the patient's bestcorrected visual acuity was 1.0 , and her intraocular pressure was $9 \mathrm{~mm} \mathrm{Hg}$.

Causes of ocular granulomatous inflammation include tuberculosis, sarcoidosis, syphilis, parasites, and viruses. Together, the findings in our case meet the criteria for the diagnosis of isolated ocular sarcoidosis of the ciliary body [1].

\section{Discussion}

Ciliary body ring melanoma is a rare tumor that may present with few symptoms and often caries a poor prognosis. One reason for the poor prognosis may be a delay in making the correct diagnosis and the misdiagnosis as a refractory glaucoma. Therefore, awareness in practitioners should be high. Clinical findings are diverse and in- 

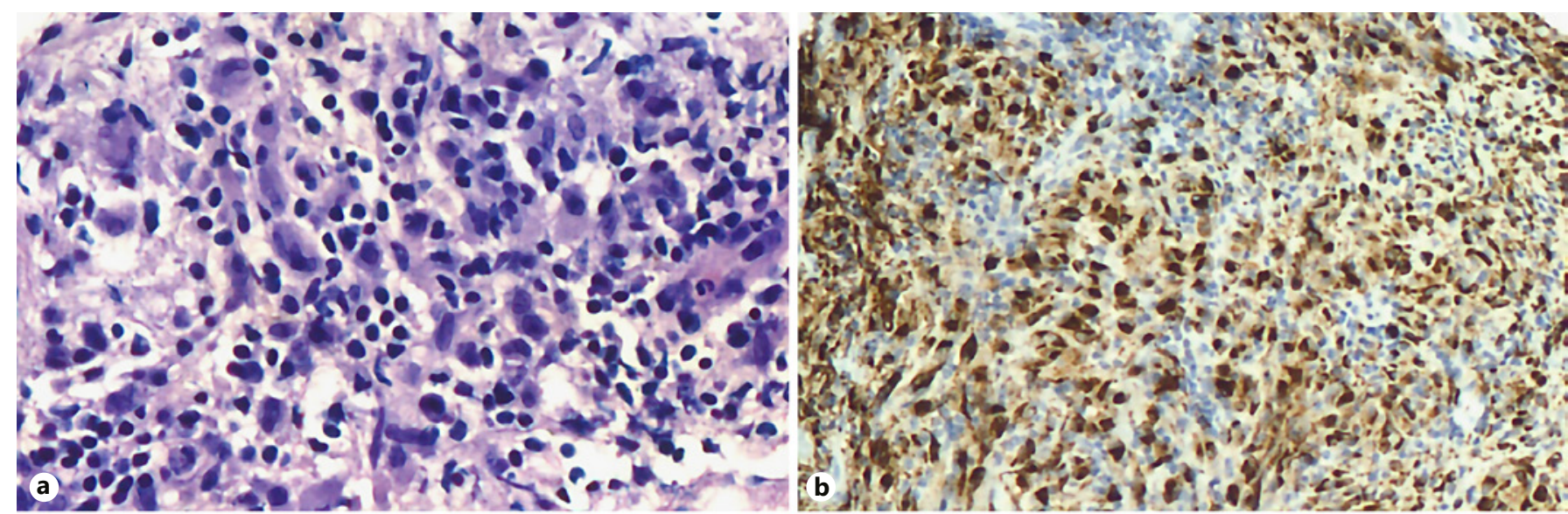

Fig. 2. Histology of the lesion and clinical findings after treatment. Transscleral biopsy revealed a non-necrotizing granulomatous inflammation (H\&E) (a) demonstrating many CD68-(epitheloid cells) positive cells using immunohistochemistry (b). Two months after biopsy and subtenon administration of triamcinolone, the tumor had disappeared (c).

clude inflammation of the anterior chamber, increased intraocular pressure, amelanosis of the tumor with prominent vessels, cataract, and a shallow anterior chamber [2-4]. In general, uveal melanomas demonstrate a blockage on transillumination, low-to-medium internal reflectivity on ultrasonography, and a high signal on T1- and a low signal on T2-weighted MRI images.

It is well-known that systemic sarcoidosis can present with anterior segment granulomas [5]. Ocular sarcoidosis mimicking amelanotic melanoma has previously been described, but this is very rare [6]. Further, we are not aware of cases demonstrating the same extent of features suggestive of ring melanoma as the present case. The negative systemic work-up made the case particularly challenging. Due to the poor prognosis of ring melanoma, a conservative, observational approach with or without anti-inflammatory treatment would not have been sound. Awareness of both ring melanoma and sarcoidosis of the ciliary body is important, nonetheless, we believe it is essential to do a biopsy before destructive surgery is carried out.

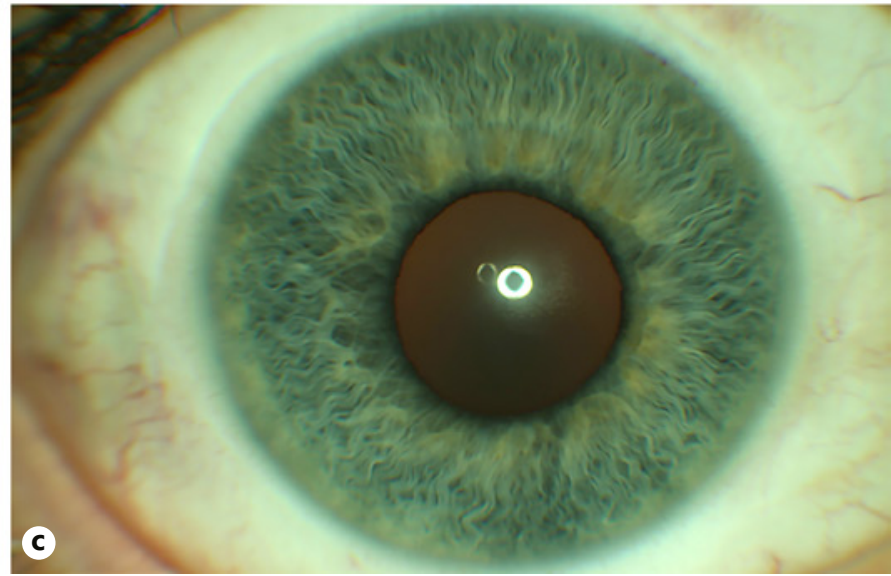

\section{Statement of Ethics}

All procedures carried out were in accordance with the tenets of the Declaration of Helsinki. Regional Ethics Committee approval is not required for a case report. The patient has given written informed consent for the publication of this case including images used.

\section{Disclosure Statement}

The authors have no conflicts of interest to declare.

\section{Funding Sources}

No funding was obtained.

\section{Author Contributions}

C.F. and J.F.K. collected data, conceived the study, analyzed data, and wrote the manuscript. R.E., K.M., S.H., and D.S. collected and analyzed data and contributed to the final version of the manuscript. 


\section{References}

1 Herbort CP, Rao NA, Mochizuki M; members of Scientific Committee of First International Workshop on Ocular Sarcoidosis. International criteria for the diagnosis of ocular sarcoidosis: results of the first International Workshop On Ocular Sarcoidosis (IWOS). Ocul Immunol Inflamm. 2009 May-Jun;17(3):160-9.

2 Demirci H, Shields CL, Shields JA, Honavar SG, Eagle RC Jr: Ring melanoma of the ciliary body: report on twenty-three patients. Retina. 2002 Dec;22(6):698-706; quiz 852-3.
3 Aziz HA, Modi YS, Plesec TP, Singh AD. Amelanotic Irido-Ciliary Ring Melanoma: A Clinicopathological Correlation. Ocul Oncol Pathol. 2016 Apr;2(3):153-5.

4 Zhao M, Mu Y, Dang Y, Zhu Y. Secondary glaucoma as initial manifestation of ring melanoma: a case report and review of literature. Int J Clin Exp Pathol. 2014 Oct;7(11):8163-9.
5 Rejdak R, Pogorelov P, Mardin CY, Szkaradek M, Juenemann AG. Solitary sarcoid granuloma of the iris mimicking tuberculosis: a case report. J Ophthalmol. 2014;2014:656042.

6 Maca SM, Firbas U, Hauff W, Zettinig G, Horvat R, Barisani-Asenbauer T. Semicircular tumor of the iris and uveitis as unilocal manifestation of sarcoidosis. Ocul Immunol Inflamm. 2004 Sep;12(3):237-40. 